University of Texas at El Paso

ScholarWorks@UTEP

$11-2014$

\title{
Is the World Itself Fuzzy? Physical Arguments for - and Unexpected Computational Consequences of - Zadeh's Vision
}

Vladik Kreinovich

The University of Texas at El Paso, vladik@utep.edu

Olga Kosheleva

The University of Texas at El Paso, olgak@utep.edu

Follow this and additional works at: https://scholarworks.utep.edu/cs_techrep

Part of the Computer Engineering Commons

Comments:

Technical Report: UTEP-CS-14-64a

To appear in: Dan E. Tamir, David Rishe, and Abraham Kandel (eds.), Fifty Years of Fuzzy Logic and Its Applications, Springer-Verlag, Berlin, Heidelberg, 2015.

\section{Recommended Citation}

Kreinovich, Vladik and Kosheleva, Olga, "Is the World Itself Fuzzy? Physical Arguments for -- and Unexpected Computational Consequences of -- Zadeh's Vision" (2014). Departmental Technical Reports (CS). 883.

https://scholarworks.utep.edu/cs_techrep/883

This Article is brought to you for free and open access by the Computer Science at ScholarWorks@UTEP. It has been accepted for inclusion in Departmental Technical Reports (CS) by an authorized administrator of ScholarWorks@UTEP. For more information, please contact Iweber@utep.edu. 


\title{
Is the World Itself Fuzzy? Physical Arguments for-and Unexpected Computational Consequences of-Zadeh's Vision
}

\author{
Vladik Kreinovich and Olga Kosheleva \\ University of Texas at El Paso \\ El Paso, Texas 79968, USA \\ vladik@utep.edu,olgak@utep.edu
}

\begin{abstract}
Fuzzy methodology has been invented to describe imprecise ("fuzzy") human statements about the world, statements that use imprecise words from natural language like "small" or "large". Usual applications of fuzzy techniques assume that the world itself is "crisp", that there are exact equations describing the world, and fuzziness of our statements is caused by the incompleteness of our knowledge. But what if the world itself is fuzzy? What if there is no perfect system of equations describing the physical world - in the sense that no matter what system of equations we try, there will always be cases when this system leads to wrong predictions? This is not just a speculation: this idea is actually supported by many physicists. At first glance, this is a pessimistic idea: no matter how much we try, we will never be able to find the the Ultimate Theory of Everything. But it turns out that this idea also has its optimistic aspects: namely, in this chapter, we show (somewhat unexpectedly), that if such a no-perfect-theory principle is true, then the use of physical data can drastically enhance computations.
\end{abstract}

\section{Fuzzy Techniques: The Original Zadeh's Vision}

Pre-Zadeh attitude: everything can be made precise. Scientists and engineers use both formal languages and an imprecise natural language. In engineering practice, formulas, derivations, and computations - which are described in a precise language - intertwine with explanations - which are usually described in a natural language. Even in formal mathematics, when presenting a proof, a mathematician describes part of it in precise terms and part in imprecise terms from a natural language: "one can easily see that", "since $\varepsilon$ is small, the difference $f(x+\varepsilon)-f(x)$ is also small", etc.

In formal mathematics, usually, the imprecise parts can be reformulated in precise terms; professional mathematicians can do it, mathematics students are taught how to do it - and math students do not get good grades until they are able to perform such a reformulation. In rare occasions, an attempt for such a formalization reveals a gap in the proof, but in most such cases, this gap is later filled. 
Similarly, when an engineer makes an imprecise argument, it does not necessarily mean that a more precise explanation is not possible: when needed, an engineer can usually provide a precise quantitative justification of his/her original qualitative decision.

A similar precisiation is often possible beyond science and engineering. For example, instructors who grade students' work use seemingly imprecise words like "excellent", "good", "satisfactory". However, in most cases, these words have a very precise meaning. In the US grading system, we usually add up well-defined points that the students got for different problems on the test. If the resulting grade is 90 (or higher) out of 100 possible points, we assign the grade "excellent" (A). If the resulting grade is at least 80 but smaller than 90 , we consider this work "good" (grade B), etc.

Similarly, in medicine, many terms that are, at first glance, imprecise, have a very precise meaning. "High blood pressure" means upper blood pressure above 140, "fever" means temperature above $37.5 \mathrm{C}$, "overweight" means that the body-mass index (body mass in $\mathrm{kg}$ divided by the squared height in meters) is above 25 , etc. In law, a child - a seemingly informal notion, with an imprecise transition - is legally defined as someone younger than 18 years old.

These example led scientists and engineers to conclude that in principle, all the statements can be made precise. According to this belief, when a statement sounds imprecise, it is only because we have not learned the corresponding terms yet. Once we learn these terms, the statement will become very precise.

Zadeh's vision. In 1965, Lotfi Zadeh published his revolutionary paper, in which he emphasized that:

- in addition to situations when use imprecise terms but have a precise meaning in mind ("excellent test results" meaning 90+ points),

- there are also many situations when we use imprecise terms for which no precise meaning is known.

Moreover, he showed that such situations, in which no precise meaning is known, in which the meaning is "fuzzy", are ubiquitous in many application areas.

To deal with such situations, L. Zadeh proposed techniques - which he called fuzzy - that enable researchers to describe their imprecise statements in precise mathematical terms, and thus, enables computer-based systems to process such statements. These techniques has led to many successful applications; see, e.g., $[3,5,6,9,16,20,22,23]$.

\section{Is the World Itself Fuzzy? And If Yes, What Are Possible Physical and Computational Consequences?}

Traditional viewpoint. The traditional viewpoint in engineering and science is that the world itself is crisp, it is described by precise equations which, in principle, enable us to predict either the events themselves (in classical, pre-quantum 
physics) or probabilities of different events (in quantum physics). The only reason for "fuzzy" uncertainty is that we only have partial knowledge about the world.

For example, when a meteorologist makes a "fuzzy" statement that there is a good chance of rain, the meteorologist usually believes that with more information, he/she would be able to make a more definite prediction.

But what if the world itself is fuzzy? But what if there are no ultimate equations? What if, no matter what equations we formulate, no matter how accurate their predictions are so far, there will always be cases when these equations will lead to wrong predictions?

In other words, what if not only our knowledge is fuzzy, what if the world itself is fuzzy?

Somewhat surprisingly, this is what many physicists actually believe. Many physicists indeed believe that every physical theory is approximate - no matter how sophisticated a theory, no matter how accurate its current predictions, inevitably new observations will surface which would require a modification of this theory; see, e.g., [2].

This belief can be justified by the history of physics: no matter how good a physical theory, no matter how good its accordance with observations, eventually, new observations appeared which were not fully consistent with the original theory - and thus, a theory needed to be modified. For example, for several centuries, Newtonian physics seems to explain all observable facts - until later, quantum (and then relativistic) effects were discovered which required changes in physical theories.

At first glance, this belief is pessimistic. This belief sounds pessimistic: no matter how much we try, we will never find the Ultimate Theory of Everything.

But maybe there is room for optimism. But is the situation indeed so pessimistic? After all, physics is not just about finding equations. Finding equations is an important first step, but the ultimate goal of physics is not to find equations, but to predict future events - and equations are an important first step towards this prediction.

Many physical equations are very complex, solving them is a complex computational task. From this viewpoint, any possibility to enhance computations would be a great optimistic development. For example, quantum physics is clearly more pessimistic in terms of possibility of predictions, because in quantum physics, we can often only predict probabilities of future events, and not the events themselves. On the other hand, research on quantum computing has shown that the use of quantum effects can drastically enhance computations; see, e.g., [17].

How does the no-perfect-theory belief affect computations? In this chapter, we analyze how the no-perfect-theory belief affects our computational abilities.

At first glance, the fact that no theory is perfect seems to make the question of computability rather hopeless: no matter how seriously we analyze computability 
within a given physical theory, eventually, this theory will turn out to be, strictly speaking, false - and thus, our analysis of what is computable will have to be redone.

In this chapter, we show, however, that in spite of this seeming hopelessness, some important answers to the question of what is computable can be deduced simply from the fact no physical theory is perfect - namely, in this case, we show that computations can be enhanced in comparison with the usual (Turing machine) computability.

Comment. Some preliminary results from this chapter first appeared in $[7,8,12$, 25].

\section{How to Describe, in Precise Terms, that No Physical Theory Is Perfect}

Discussion. The statement that no physical theory is perfect means that no matter what physical theory we have, eventually there will be observations which violate this theory. To formalize this statement, we need to formalize what are observations and what is a theory.

What are observations? Each observation can be represented, in the computer, as a sequence of $0 \mathrm{~s}$ and $1 \mathrm{~s}$; actually, in many cases, the sensors already produce the signal in the computer-readable form, as a sequence of $0 \mathrm{~s}$ and $1 \mathrm{~s}$.

An exact description of each experiment can also be described in precise terms, and thus, it will be represented in a computer as a sequence of $0 \mathrm{~s}$ and $1 \mathrm{~s}$. An experiment should specify how long we wait for the result; in this way, we are guaranteed that we get the result. The coding should be done in such a way that the waiting time does not exceed a polynomial of the length of the code $i$; for example, if we want to wait for $t$ moments of time, we should just add $t$ copies of an appropriate wait symbol.

In each experiment, we can specify which bit of the result we are interested in; for convenience, we can consider producing different bits as different experiments.

Each such experiment is represented as a sequence of $0 \mathrm{~s}$ and $1 \mathrm{~s}$; by appending 1 at the beginning of this sequence, we can view this sequence as a binary expansion of a natural number $i$. This natural number will serve as the "code" describing the experiment. For example, a sequence 001 is transformed into $i=$ $1001_{2}=9_{10}$. (We need to append 1 , because otherwise two different sequences 001 and 01 will be represented by the same integer).

For natural numbers $i$ which correspond to experiment descriptions, let $\omega_{i}$ denote the bit result of the experiment described by the code $i$.

Let us also define $\omega_{i}$ for natural numbers $i$ which do not correspond to a syntactically correct description of experiments. For example, we can take $\omega_{i}=0$ for such numbers $i$.

In these terms, all past and future observations form a (potentially) infinite sequence $\omega=\omega_{1} \omega_{2} \ldots$ of $0 \mathrm{~s}$ and $1 \mathrm{~s}, \omega_{i} \in\{0,1\}$. 
What is a physical theory from the viewpoint of our problem: a set of sequences. A physical theory may be very complex, but all we care about is which sequences of observations $\omega$ are consistent with this theory and which are not. In other words, for our purposes, we can identify a physical theory $T$ with the set of all sequences $\omega$ which are consistent with this theory.

Not every set of sequences corresponds to a physical theory: the set $T$ must be non-empty and definable. Not every set of sequences comes from a physical theory. First, a physical theory must have at least one possible sequence of observations, i.e., the set $T$ must be non-empty.

Second, a theory - and thus, the corresponding set - must be described by a finite sequence of symbols in an appropriate language. Sets which are uniquely by (finite) formulas are known as definable. Thus, the set $T$ must be definable.

Since at any moment of time, we only have finitely many observations, the set $T$ must be closed. Another property of a physical theory comes from the fact that at any given moment of time, we only have finitely many observations, i.e., we only observe finitely many bits. From this viewpoint, we say that observations $\omega_{1} \ldots \omega_{n}$ are consistent with the theory $T$ if there is a continuing infinite sequence which is consistent with this theory, i.e., which belongs to the set $T$.

The only way to check whether an infinite sequence $\omega=\omega_{1} \omega_{2} \ldots$ is consistent with the theory is to check that for every $n$, the sequences $\omega_{1} \ldots \omega_{n}$ are consistent with the theory $T$. In other words, we require that for every infinite sequence $\omega=\omega_{1} \omega_{2} \ldots$,

- if for every $n$, the sequence $\omega_{1} \ldots \omega_{n}$ is consistent with the theory $T$, i.e., if for every $n$, there exists a sequence $\omega^{(n)} \in T$ which has the same first $n$ bits as $\omega$, i.e., for which $\omega_{i}^{(n)}=\omega_{i}$ for all $i=1, \ldots, n$,

- then the sequence $\omega$ itself should be consistent with the theory, i.e., this infinite sequence should also belong to the set $T$.

From the mathematical viewpoint, we can say that the sequences $\omega^{(n)}$ converge to $\omega: \omega^{(n)} \rightarrow \omega$ (or, equivalently, $\lim \omega^{(n)}=\omega$ ), where convergence is understood in terms of the usual metric on the set of all infinite sequences $d\left(\omega, \omega^{\prime}\right) \stackrel{\text { def }}{=}$ $2^{-N\left(\omega, \omega^{\prime}\right)}$, where $N\left(\omega, \omega^{\prime}\right) \stackrel{\text { def }}{=} \max \left\{k: \omega_{1} \ldots \omega_{k}=\omega_{1}^{\prime} \ldots \omega_{k}^{\prime}\right\}$.

In general, if $\omega^{(m)} \rightarrow \omega$ in the sense of this metric, this means that for every $n$, there exists an integer $\ell$ such that for every $m \geq \ell$, we have $\omega_{1}^{(m)} \ldots \omega_{n}^{(m)}=$ $\omega_{1} \ldots \omega_{n}$. Thus, if $\omega^{(m)} \in T$ for all $m$, this means that for every $n$, a finite sequence $\omega_{1} \ldots \omega_{n}$ can be a part of an infinite sequence which is consistent with the theory $T$. In view of the above, this means that $\omega \in T$.

In other words, if $\omega^{(m)} \rightarrow \omega$ and $\omega^{(m)} \in T$ for all $m$, then $\omega \in T$. So, the set $T$ must contain all the limits of all its sequences. In topological terms, this means that the set $T$ must be closed.

A physical theory must be different from a fact and hence, the set $T$ must be nowhere dense. The assumption that we are trying to formalize is that no matter how many observations we have which confirm a theory, there eventually will 
be a new observation which is inconsistent with this theory. In other words, for every finite sequence $\omega_{1} \ldots \omega_{n}$ which is consistent with the set $T$, there exists a continuation of this sequence which does not belong to $T$. The opposite would be if all the sequences which start with $\omega_{1} \ldots \omega_{n}$ belong to $T$; in this case, the set $T$ will be dense in this set. Thus, in mathematical terms, the statement that every finite sequence which is consistent with $T$ has a continuation which is not consistent with $T$ means that the set $T$ is nowhere dense.

Resulting definition of a theory. By combining the above properties of a set $T$ which describes a physical theory, we arrive at the following definition.

Definition 1. By a physical theory, we mean a non-empty closed nowhere dense definable set $T$.

Mathematical comment. To properly define what is definable, we need to have a consistent formal definition of definability. In this chapter, we follow a natural definition from $[10,11]$ - which is reproduced in Appendix A.

Formalization of the principle that no physical theory is perfect. In terms of the above notations, the no-perfect-theory principle simply means that the infinite sequence $\omega$ (describing the results of actual observations) is not consistent with any physical theory, i.e., that the sequence $\omega$ does not belong to any physical theory $T$. Thus, we arrive at the following definition.

Definition 2. We say that an infinite binary sequence $\omega$ is consistent with the no-perfect-theory principle if the sequence $\omega$ does not belong to any physical theory (in the sense of Definition 1).

Comment. Are there such sequences in the first place? Our answer is yes. Indeed, by definition, we want a sequence which does not belong to a union of all definable physical theories. Every physical theory is closed nowhere dense set. Every definable set is defined by a finite sequence of symbols, so there are no more than countably many definable theories. Thus, the union of all definable physical theories is contained in a union of countably many closed nowhere dense sets. Such sets are knows as meager (or Baire first category); it is known that the set of all infinite binary sequences is not meager. Thus, there are sequences who do not belong to the above union - i.e., sequences which are consistent with the no-perfect-theory principle; see, e.g., $[4,18]$.

\section{How to Describe When Access to Physical Observations Enhances Computability}

How to describe general computations. Each computation is a solution to a welldefined problem. As a result, each bit in the resulting answer satisfies a welldefined mathematical property. All mathematical properties can be described, e.g., in terms of Zermelo-Fraenkel theory ZF, the standard formalization of set theory. For each resulting bit, we can formulate a property $P$ which is true if and only if this bit is equal to 1 . In this sense, each bit in each computation result 
can be viewed as the truth value of some statement formulated in ZF. Thus, our general ability to compute can be described as the ability to (at least partially) compute the sequence of truth values of all statements from ZF.

All well-defined statements from ZF can be numbered, e.g., in lexicographic order. Let $\alpha_{n}$ denote the truth value of the $n$-th ZF statement, and let $\alpha=$ $\alpha_{1} \ldots \alpha_{n} \ldots$ denote the infinite sequence formed by these truth values. In terms of this sequence, our ability to compute is our ability to compute the sequence $\alpha$.

Kolmogorov complexity as a way to describe what is easier to compute. We want to analyze whether the use of physical observations (i.e., of the sequence $\omega$ analyzed in the previous section) can simplify computations. A natural measure of easiness-to-compute was invented by A. N. Kolmogorov, the founder of modern probability theory, when he realized that in the traditional probability theory, there is no formal way to distinguish between:

- finite sequences which come from observing from truly random processes, and

- orderly sequences like $0101 \ldots 01$.

Kolmogorov noticed that an orderly sequence $0101 \ldots 01$ can be computed by a short program, while the only way to compute a truly random sequence $0101 \ldots$ is to have a print statement that prints this sequence. He suggested to describe this difference by introducing what is now known as Kolmogorov complexity $K(x)$ of a finite sequence $x$ : the shortest length of a program (in some programming language) which computes the sequence $x$.

- For an orderly sequence $x$, the Kolmogorov complexity $K(x)$ is much smaller than the length len $(x)$ of this sequence: $K(x) \ll \operatorname{len}(x)$.

- For a truly random sequence $x$, we have $K(x) \approx \operatorname{len}(x)$; see, e.g., [14].

The smaller the difference len $(x)-K(x)$, the more we are sure that the sequence $x$ is truly random.

Relative Kolmogorov complexity as a way to describe when using an auxiliary sequence simplifies computations. The usual notion of Kolmogorov complexity provides the complexity of computing $x$ "from scratch". A similar notion of the relative Kolmogorov complexity $K(x \mid y)$ can be used to describe the complexity of computing $x$ when a (potentially infinite) sequence $y$ is given. This relative complexity is based on programs which are allowed to use $y$ as a subroutine, i.e., programs which, after generating an integer $n$, can get the $n$-th bit $y_{n}$ of the sequence $y$ by simply calling $y$. When we compute the length of such programs, we just count the length of the parameters of this call, not the length of the auxiliary program which computes $y_{n}$ - just like when we count the length of a $\mathrm{C}++$ program, we do not count how many steps it takes to compute, e.g., $\sin (x)$, we just count the number of symbols in this function call. The relative Kolmogorov complexity is then defined as the shortest length of such a $y$-using program which computes $x$. 
Clearly, if $x$ and $y$ are unrelated, having access to $y$ does not help in computing $x$, so $K(x \mid y) \approx K(x)$. On the other hand, if $x$ coincides with $y$, then the relative complexity $K(x \mid y)$ is very small: all we need is a simple for-loop, in which we call for each bit $y_{i}, i=1, \ldots, n$, and print this bit right away.

Resulting reformulation of our question. In terms of relative Kolmogorov complexity, the question of whether observations enhance computations is translated into checking whether $K\left(\alpha_{1} \ldots \alpha_{n} \mid \omega\right) \approx K\left(\alpha_{1} \ldots \alpha_{n}\right)$ (in which case there is no enhancement) or whether $K\left(\alpha_{1} \ldots \alpha_{n} \mid \omega\right) \ll K\left(\alpha_{1} \ldots \alpha_{n}\right)$ (in which case there is a strong enhancement). The larger the difference $K\left(\alpha_{1} \ldots \alpha_{n}\right)-K\left(\alpha_{1} \ldots \alpha_{n} \mid \omega\right)$, the larger the enhancement.

\section{$5 \quad$ First Result: No-Perfect-Theory Principle Enhances Computability}

Let us show that under the no-perfect-theory principle, observations do indeed enhance computability.

Proposition 1. Let $\alpha$ be a sequence of truth values of ZF statements, and let $\omega$ be an infinite binary sequence which is consistent with the no-perfect-theory principle. Then, for every integer $C>0$, there exists an integer $n$ for which $K\left(\alpha_{1} \ldots \alpha_{n} \mid \omega\right)<K\left(\alpha_{1} \ldots \alpha_{n}\right)-C$.

In other words, in principle, we can have an arbitrary large enhancement.

Comment. For readers' convenience, all the proof are placed in a special appendix.

\section{Can Access to Physical Observations Speed Up Computations?}

Are computations feasible? What we have shown so far is that under Zadehinspired no-perfect-theory belief, it is possible to compute things that are not computable in the usual physical paradigm. From the practical viewpoint, being able to compute something in principle is important, but even more important is how fast we can compute it. In many cases, computations are theoretically possible, but not practically feasible, since they require computation times which are longer than the lifetime of the Universe :-( It is therefore important to analyze which problems are feasibly computable and which are not. To perform this analysis, we need to define what is "feasible" and what is a "problem".

In computer science, "feasible" is usually interpreted as computable in polynomial time, i.e., in time $t$ bounded by a polynomial of the length $n$ of the input; see, e.g., [19]. This definition works in most cases:

- time $2^{n}$ is non-feasible already for $n \approx 300$, while

- time $n^{2}$ or $n^{3}$ is usually feasible. 
This is not a perfect definition:

- on the one hand, time $t=10^{400} \cdot n$ is polynomial in $n$ but clearly not feasible;

- on the other hand, computation time $\exp \left(10^{-10} \cdot n\right)$ is not bounded by a polynomial, but it clearly corresponds to feasible computations.

However, this is the best definition we have.

By a problem, computer scientists usually understand a problem in which it is absolutely clear what is a solution and what is not. For example:

- finding a proof of a given mathematical statement,

- finding a formula that fits all experimental observations,

- designing a bridge under certain specifications of strength, cost, etc.,

these are all such problems - while, e.g., the problem of designing a beautiful bridge is not clearly defined.

In general, we need to find a solution that satisfies a given set of constraints - or at least check that such a solution is possible. Once we have a candidate for the solution, we can feasibly check whether this candidate indeed satisfies all the constraints.

A problem of checking whether a given set of constraints has solution is called a problem of the class NP if we can check, in polynomial time, whether a given candidate is a solution; see, e.g., [19].

Examples of such problem includes checking whether a given graph can be colored in 3 colors, checking whether a given propositional formula - i.e., formula of the type

$$
\left(v_{1} \vee \neg v_{2} \vee v_{3}\right) \&\left(v_{4} \vee \neg v_{2} \vee \neg v_{5}\right) \& \ldots,
$$

is satisfiable, i.e., whether this formula is true by some combination of the propositional variables $v_{i}$, etc.

Each problem from the class NP can be algorithmically solved by trying all possible candidates. For example, we can check whether a graph can be colored by trying all possible assignments of colors to different vertices of a graph, and we can check whether a given propositional formula is satisfiable by trying all $2^{n}$ possible combinations of true-or-false values $v_{1}, \ldots, v_{n}$. Such exhaustive search algorithms require computation time like $2^{n}$, time that grows exponentially with $n$. For medium-size inputs, e.g., for $n \approx 300$, the resulting time is larger than the lifetime of the Universe. So, these exhaustive search algorithms are not practically feasible.

It is not known whether problems from the class NP can be solved feasibly (i.e., in polynomial time): this is the famous open problem $\mathrm{P} \stackrel{?}{=} \mathrm{NP}$. It is known, however, there are problems in the class NP which are NP-complete in the sense that every problem from the class NP can be reduced to this problem. Reduction means, in particular, that if we can find a way to efficiently solve one NPcomplete problem, then, by reducing other problems from the class NP to this problem, we can thus efficiently solve all the problems from the class NP.

So, it is very important to be able to efficiently solve even one NP-hard problem. (By the way, both above example of NP problems - checking whether 
a graph can be colored in 3 colors and whether coloring a propositional formula is satisfiable - are NP-complete.)

Can the use of non-standard physics speed up the solution of NP-complete problems? NP-completeness of a problem means, crudely speaking, that the problem may take an unrealistically long time to solve - at least on computers based on the usual physical techniques. A natural question is: can the use of non-standard physics speed up the solution of these problems?

This question has been analyzed for several specific physical theories, e.g., for quantum filed theory, for cosmological solutions with wormholes and/or casual anomalies. Several possible techniques for solving NP-complete problems are described in $[1,11,13,15,21]$.

How does the no-perfect-belief affect the speed of computations? In this chapter, we show that an important speed-up can be deduced simply from the fact no physical theory is perfect.

\section{Second Result: The Use of Physical Observations Can Help in Solving NP-Complete Problems}

How to represent instances of an NP-complete problem. For each NP-complete problem $\mathcal{P}$, its instances are sequences of symbols. In the computer, each such sequence is represented as a sequence of $0 \mathrm{~s}$ and $1 \mathrm{~s}$. Thus, as in the previous sections, we can append 1 in front of this sequence and interpret the resulting sequence as a binary code of a natural number $i$.

In principle, not all natural numbers $i$ correspond to instances of a problem $\mathcal{P}$; we will denote the set of all natural numbers which correspond to such instances by $S_{\mathcal{P}}$.

For each $i \in S_{\mathcal{P}}$, the correct answer (true or false) to the $i$-th instance of the problem $\mathcal{P}$ will be denoted by $s_{\mathcal{P}, i}$.

What we mean by using physical observations in computations. In addition to performing computations, our computational device can produce a scheme $i$ for an experiment, and then use the result $\omega_{i}$ of this experiment in future computations. In other words, given an integer $i$, we can produce $\omega_{i}$.

In precise theory-of-computation terms, the use of physical observations in computations thus means computations that use the sequence $\omega$ as an oracle; see, e.g., [19].

Definition 3. By a ph-algorithm $\mathcal{A}$, we mean an algorithm which uses, as an oracle, a sequence $\omega$ which is consistent with the no-perfect-theory principle.

Notation. The result of applying an algorithm $\mathcal{A}$ using $\omega$ to an input $i$ will be denoted by $\mathcal{A}(\omega, i)$.

Definition 4. Let $\mathcal{P}$ be an NP-complete problem. We say that a feasible phalgorithm $\mathcal{A}$ solves almost all instances of $\mathcal{P}$ if for every $\varepsilon>0$, and for every natural number $n$, there exists an integer $N \geq n$ for which the proportion of the 
instances $i \leq N$ of the problem $\mathcal{P}$ which are correctly solved by $\mathcal{A}$ is greater than $1-\varepsilon$ :

$$
\forall \varepsilon>0 \forall n \exists N\left(N \geq n \& \frac{\#\left\{i \leq N: i \in S_{\mathcal{P}} \& \mathcal{A}(\omega, i)=s_{\mathcal{P}, i\}}\right.}{\#\left\{i \leq N: i \in S_{\mathcal{P}}\right\}}>1-\varepsilon\right) .
$$

Comment. The restriction to sufficiently long inputs $N \geq n$ makes perfect sense: for short inputs, NP-completeness is not an issue: we can perform exhaustive search of all possible bit sequences of length 10,20, and even 30 . The challenge starts when the length of the input is high.

Proposition 2. For every NP-complete problem $\mathcal{P}$, there exists a feasible phalgorithm $\mathcal{A}$ that solves almost all instances of $\mathcal{P}$.

In other words, we show that the use of physical observations makes all NPcomplete problems easier-to-solve (in the above-described sense).

Acknowledgments. This work was supported in part by the National Science Foundation grants HRD-0734825, HRD-124212, and DUE-0926721. The authors are thankful to the anonymous referees for valuable suggestions.

\section{References}

1. Aaronson, S.: NP-complete problems and physical reality. ACM SIGACT News 36, 30-52 (2005)

2. Feynman, R., Leighton, R., Sands, M.: The Feynman Lectures on Physics, Addison Wesley, Boston, Massachusetts (2005)

3. Friedman, M., Kandel, A.: Introduction to Pattern Recognition: Statistical, Structural, Neural and Fuzzy Logic Approaches, World Scientific, Singapore (1999)

4. Jalal-Kamali, A., Nebesky, O., Durcholz, M.H., Kreinovich, V., Longpré, L.: Towards a "generic" notion of genericity: from "typical" and "random" to meager, shy, etc. Journal of Uncertain Systems 6(2), 104-113 (2012)

5. Kandel, A. Fuzzy Expert Systems, CRC Press, Boca Raton, Florida (1991)

6. Kandel, A., Langholz, G.: Fuzzy Control Systems, CRC Press, Boca Raton, Florida (1993)

7. Kosheleva, O.M., Soloviev, S.V.: On the logic of using observable events in decision making. In: Proceedings of the IX National USSR Symposium on Cybernetics, Moscow, 1981, pp. 49-51, in Russian (1981)

8. Kosheleva, O., Zakharevich, M., Kreinovich, V.: If many physicists are right and no physical theory is perfect, then by using physical observations, we can feasibly solve almost all instances of each NP-complete problem. Mathematical Structures and Modeling, to appear.

9. Klir G., Yuan, B.: Fuzzy Sets and Fuzzy Logic: Theory and Applications, Upper Saddle River, New Jersey: Prentice Hall (1995)

10. Kreinovich, V,: Toward formalizing non-monotonic reasoning in physics: the use of Kolmogorov complexity. Revista Iberoamericana de Inteligencia Artificial 41, 4-20(2009)

11. Kreinovich, V., Finkelstein, A.M.: Towards applying computational complexity to foundations of physics. Journal of Mathematical Sciences 134(5), 2358-2382 (2006) 
12. Kreinovich, V., Kosheleva, O.: Logic of scientific discovery: how physical induction affects what is computable. In: Proceedings of the The International Interdisciplinary Conference Philosophy, Mathematics, Linguistics: Aspects of Interaction 2014 PhML'2014, St. Petersburg, Russia, April 21-25, 2014, pp. 116-127 (2014)

13. Kreinovich, V., Margenstern, M.: In some curved spaces, one can solve NP-hard problems in polynomial time. Notes of Mathematical Seminars of St. Petersburg Department of Steklov Institute of Mathematics 358, 224-250 (2008); reprinted in Journal of Mathematical Sciences 158(5), 727-740 (2009)

14. Li, M., Vitányi, P.M.B.: An Introduction to Kolmogorov Complexity, SpringerVerlag, Berlin, Hiedelberg, New York (2008)

15. Morgenstein, D., Kreinovich, V.: Which algorithms are feasible and which are not depends on the geometry of space-time. Geombinatorics 4(3), 80-97 (1995)

16. Nguyen, H.T., Walker, E.A.: First Course on Fuzzy Logic, CRC Press, Boca Raton, Florida (2006)

17. Nielsen, M.A., Chuang, I.L.: Quantum Computation and Quantum Information, Cambridge University Press, Cambridge, Massachusetts (2011)

18. Oxtoby, J.C.: Measure and Category: A Survey of the Analogies between Topological and Measure Spaces, Springer Verlag, New York, Heidelberg, Berlin (1980)

19. Papadimitriou, C.: Computational Complexity, Addison Welsey, Reading, Massachusetts (1994)

20. Ross, T.J.: Fuzzy Logic with Engineering Applications, Wiley, New York (2010)

21. Srikanth, R.: The quantum measurement problem and physical reality: a computation theoretic perspective, in: Goswami, D. (ed.), Quantum Computing: Back Action 2006, IIT Kanpur, India, March 2006, AIP Conference Proceedings, Vol. 864, pp. 178-193 (2006)

22. Terano, T., Asai, K., Sugeno, M. (Eds): Fuzzy Systems Theory and Its Applications, Academic Press, San Diego, California (1992)

23. Teodorescu, H.-N.L., Kandel, A., Jain, L.C.: Fuzzy and Neuro-Fuzzy Systems in Medicine, CRC Press, Boca Raton, Florida (1998)

24. Zadeh, L.A.: Fuzzy sets, Information and Control 8, 338-353 (1965)

25. Zakharevich, M., Kosheleva, O.: If many physicists are right and no physical theory is perfect, then the use of physical observations can enhance computations. Journal of Uncertain Systems 8(3), 227-232 (2014)

\section{A A Formal Definition of Definable Sets}

Definition A1. Let $\mathcal{L}$ be a theory, and let $P(x)$ be a formula from the language of the theory $\mathcal{L}$, with one free variable $x$ for which the set $\{x \mid P(x)\}$ is defined in the theory $\mathcal{L}$. We will then call the set $\{x \mid P(x)\} \mathcal{L}$-definable.

Crudely speaking, a set is $\mathcal{L}$-definable if we can explicitly define it in $\mathcal{L}$. The set of all real numbers, the set of all solutions of a well-defined equation, every set that we can describe in mathematical terms: all these sets are $\mathcal{L}$-definable.

This does not mean, however, that every set is $\mathcal{L}$-definable: indeed, every $\mathcal{L}$-definable set is uniquely determined by formula $P(x)$, i.e., by a text in the language of set theory. There are only denumerably many words and therefore, there are only denumerably many $\mathcal{L}$-definable sets. Since, e.g., in a standard model of set theory $\mathrm{ZF}$, there are more than denumerably many sets of integers, some of them are thus not $\mathcal{L}$-definable. 
Our objective is to be able to make mathematical statements about $\mathcal{L}$ definable sets. Therefore, in addition to the theory $\mathcal{L}$, we must have a stronger theory $\mathcal{M}$ in which the class of all $\mathcal{L}$-definable sets is a set - and it is a countable set.

Denotation. For every formula $F$ from the theory $\mathcal{L}$, we denote its Gödel number by $\lfloor F\rfloor$.

Comment. A Gödel number of a formula is an integer that uniquely determines this formula. For example, we can define a Gödel number by describing what this formula will look like in a computer. Specifically, we write this formula in $\mathrm{AT}_{\mathrm{E} X}$, interpret every $\mathrm{AT}_{\mathrm{E} X} \mathrm{X}$ symbol as its ASCII code (as computers do), add 1 at the beginning of the resulting sequence of $0 \mathrm{~s}$ and $1 \mathrm{~s}$, and interpret the resulting binary sequence as an integer in binary code.

Definition A2. We say that a theory $\mathcal{M}$ is stronger than $\mathcal{L}$ if it contains all formulas, all axioms, and all deduction rules from $\mathcal{L}$, and also contains a special predicate $\operatorname{def}(n, x)$ such that for every formula $P(x)$ from $\mathcal{L}$ with one free variable, the formula $\forall y(\operatorname{def}(\lfloor P(x)\rfloor, y) \leftrightarrow P(y))$ is provable in $\mathcal{M}$.

The existence of a stronger theory can be easily proven: indeed, for $\mathcal{L}=\mathrm{ZF}$, there exists a stronger theory $\mathcal{M}$. As an example of such a stronger theory, we can simply take the theory $\mathcal{L}$ plus all countably many equivalence formulas as described in Definition A2 (corresponding to all possible formulas $P(x)$ with one free variable). This theory clearly contains $\mathcal{L}$ and all the desired equivalence formulas, so all we need to prove is that the resulting theory $\mathcal{M}$ is consistent (provided that $\mathcal{L}$ is consistent, of course). Due to compactness principle, it is sufficient to prove that for an arbitrary finite set of formulas $P_{1}(x), \ldots, P_{m}(x)$, the theory $\mathcal{L}$ is consistent with the above reflection-principle-type formulas corresponding to these properties $P_{1}(x), \ldots, P_{m}(x)$.

This auxiliary consistency follows from the fact that for such a finite set, we can take

$$
\operatorname{def}(n, y) \leftrightarrow\left(n=\left\lfloor P_{1}(x)\right\rfloor \& P_{1}(y)\right) \vee \ldots \vee\left(n=\left\lfloor P_{m}(x)\right\rfloor \& P_{m}(y)\right)
$$

This formula is definable in $\mathcal{L}$ and satisfies all $m$ equivalence properties. The statement is proven.

Important comments. In the main text, we will assume that a theory $\mathcal{M}$ that is stronger than $\mathcal{L}$ has been fixed; proofs will mean proofs in this selected theory $\mathcal{M}$.

An important feature of a stronger theory $\mathcal{M}$ is that the notion of an $\mathcal{L}$ definable set can be expressed within the theory $\mathcal{M}$ : a set $S$ is $\mathcal{L}$-definable if and only if $\exists n \in \mathbb{N} \forall y(\operatorname{def}(n, y) \leftrightarrow y \in S)$.

In the chapter, when we talk about definability, we will mean this property expressed in the theory $\mathcal{M}$. So, all the statements involving definability become statements from the theory $\mathcal{M}$ itself, not statements from metalanguage. 


\section{B Proofs}

Proof of Proposition 1. Let us fix an integer $C$. To prove the desired property for this $C$, let us prove that the set $T$ of all the sequences which do not satisfy this property, i.e., for which $K\left(\alpha_{1} \ldots \alpha_{n} \mid \omega\right) \geq K\left(\alpha_{1} \ldots \alpha_{n}\right)-C$ for all $n$, is a physical theory in the sense of Definition 1. For this, we need to prove that this set $T$ is non-empty, closed, nowhere dense, and definable. Then, from Definition 2, it will follow that the sequence $\omega$ does not belong to this set and thus, that the conclusion of Proposition 1 is true.

The set $T$ is clearly non-empty: it contains, e.g., a sequence $\omega=00 \ldots 0 \ldots$ which does not affect computations. The set $T$ is also clearly definable: we have just defined it.

Let us prove that the set $T$ is closed. For that, let us assume that $\omega^{(m)} \rightarrow \omega$ and $\omega^{(m)} \in T$ for all $m$. We then need to prove that $\omega \in T$. Indeed, let us fix $n$, and let us prove that $K\left(\alpha_{1} \ldots \alpha_{n} \mid \omega\right) \geq K\left(\alpha_{1} \ldots \alpha_{n}\right)-C$. We will prove this by contradiction. Let us assume that $K\left(\alpha_{1} \ldots \alpha_{n} \mid \omega\right)<K\left(\alpha_{1} \ldots \alpha_{n}\right)-C$. This means that there exists a program $p$ of length $\operatorname{len}(p)<K\left(\alpha_{1} \ldots \alpha_{n}\right)-C$ which uses $\omega$ to compute $\alpha_{1} \ldots \alpha_{n}$. This program uses only finitely many bits of $\omega$; let $B$ be the largest index of these bits. Due to $\omega^{(m)} \rightarrow \omega$, there exists $M$ for which, for all $m \geq M$, the first $B$ bits of $\omega^{(m)}$ coincide with the first $B$ bits of the sequence $\omega$. Thus, the same program $p$ will work exactly the same way - and generate the same sequence $\alpha_{1} \ldots \alpha_{n}$ - if we use $\omega^{(m)}$ instead of $\omega$. But since len $(p)<K\left(\alpha_{1} \ldots \alpha_{n}\right)-C$, this would means that the shortest length $K\left(\alpha_{1} \ldots \alpha_{n} \mid \omega^{(m)}\right)$ of all the programs which use $\omega^{(m)}$ to compute $\alpha_{1} \ldots \alpha_{n}$ also satisfies the inequality $K\left(\alpha_{1} \ldots \alpha_{n} \mid \omega^{(m)}\right)<K\left(\alpha_{1} \ldots \alpha_{n}\right)-C$. This inequality contradicts to our assumption that $\omega^{(m)} \in T$ and thus, that $K\left(\alpha_{1} \ldots \alpha_{n} \mid \omega^{(m)}\right) \geq$ $K\left(\alpha_{1} \ldots \alpha_{n}\right)-C$. The contradiction proves that the set $T$ is indeed closed.

Let us now prove that the set $T$ is nowhere dense, i.e., that for every finite sequence $\omega_{1} \ldots \omega_{m}$, there exists a continuation $\omega$ which does not belong to the set $T$. Indeed, as such a continuation, we can simply take a sequence $\omega=\omega_{1} \ldots \omega_{m} \alpha_{1} \alpha_{2} \ldots$ obtained by appending $\alpha$ at the end. For this new sequence, computing $\alpha_{1} \ldots \alpha_{n}$ is straightforward: we just copy the values $\alpha_{i}$ from the corresponding places of the new sequence $\omega$. Here, the relative Kolmogorov complexity $K\left(\alpha_{1} \ldots \alpha_{n} \mid \omega\right)$ is very small and is, thus, much smaller than the complexity $K\left(\alpha_{1} \ldots \alpha_{n}\right)$ which - since ZF is not decidable - grows with $n$.

The proposition is proven.

\section{Proof of Proposition 2.}

$1^{\circ}$. As the desired ph-algorithm, we will, given an instance $i$, simply produce the result $\omega_{i}$ of the $i$-th experiment. Let us prove, by contradiction, that this algorithm satisfies the desired property.

$2^{\circ}$. We want to prove that for every $\varepsilon>0$ and for every $n$, there exists an integer $N \geq n$ for which

$$
\#\left\{i \leq N: i \in S_{\mathcal{P}} \& \omega_{i}=s_{\mathcal{P}, i}\right\}>(1-\varepsilon) \cdot \#\left\{i \leq N: i \in S_{\mathcal{P}}\right\} .
$$


The assumption that this property is not satisfied means that for some $\varepsilon>0$ and for some integer $n$, we have

$$
\#\left\{i \leq N: i \in S_{\mathcal{P}} \& \omega_{i}=s_{\mathcal{P}, i}\right\} \leq(1-\varepsilon) \cdot \#\left\{i \leq N: i \in S_{\mathcal{P}}\right\} \text { for all } N \geq n .
$$

Let $T$ denote the set of all the sequences $x$ that satisfy the property (1), i.e., let

$$
T \stackrel{\text { def }}{=}
$$

$\left\{x: \#\left\{i \leq N: i \in S_{\mathcal{P}} \& x_{i}=s_{\mathcal{P}, i}\right\} \leq(1-\varepsilon) \cdot \#\left\{i \leq N: i \in S_{\mathcal{P}}\right\}\right.$ for all $\left.N \geq n\right\}$.

We will prove that this set $T$ is a physical theory in the sense of Definition 1 .

Then, due to Definition 2 and the fact that the sequence $\omega$ satisfies the noperfect-theory principle, we will be able to conclude that $\omega \notin T$, and thus, that the property (1) is not satisfied for the given sequence $\omega$. This will conclude the proof by contradiction.

$3^{\circ}$. By definition of a physical theory $T$, it is a set which is non-empty, closed, nowhere dense, and definable. Let us prove these four properties one by one.

$3.1^{\circ}$. Non-emptiness comes from the fact that the sequence $x_{i}$ for which $x_{i}=$ $\neg s_{\mathcal{P}, i}$ for $i \in S_{\mathcal{P}}$ and $x_{i}=0$ otherwise clearly belongs to this set: for this sequence, for every $N$, we have $\#\left\{i \leq N: i \in S_{\mathcal{P}} \& x_{i}=s_{\mathcal{P}, i}\right\}=0$ and thus, the desired property is satisfied.

$3.2^{\circ}$. Let us prove that the set $T$ is closed, i.e., that if we have a family of sequences $x^{(m)} \in T$ for which $x^{(m)} \rightarrow x$, then $x \in T$.

Indeed, let us take any $N \neq n$, and let us prove that

$$
\#\left\{i \leq N: i \in S_{\mathcal{P}} \& x_{i}=s_{\mathcal{P}, i}\right\} \leq(1-\varepsilon) \cdot \#\left\{i \leq N: i \in S_{\mathcal{P}}\right\}
$$

for this $N$. Due to $x^{(m)} \rightarrow x$, there exists $M$ for which, for all $m \geq M$, the first $N$ bits of $x^{(m)}$ coincide with the first $N$ bits of the sequence $x: x_{i}^{(m)}=\omega_{i}$ for all $i \leq N$. Thus,

$$
\#\left\{i \leq N: i \in S_{\mathcal{P}} \& x_{i}=s_{\mathcal{P}, i}\right\}=\#\left\{i \leq N: i \in S_{\mathcal{P}} \& x_{i}^{(m)}=s_{\mathcal{P}, i}\right\} .
$$

Since $x^{(m)} \in T$, we have

$$
\#\left\{i \leq N: i \in S_{\mathcal{P}} \& x_{i}^{(m)}=s_{\mathcal{P}, i}\right\} \leq(1-\varepsilon) \cdot \#\left\{i \leq N: i \in S_{\mathcal{P}}\right\},
$$

thus

$$
\#\left\{i \leq N: i \in S_{\mathcal{P}} \& x_{i}=s_{\mathcal{P}, i}\right\} \leq(1-\varepsilon) \cdot \#\left\{i \leq N: i \in S_{\mathcal{P}}\right\} .
$$

So, the set $T$ is indeed closed.

$3.3^{\circ}$. Let us now prove that the set $T$ is nowhere dense, i.e., that for every finite sequence $x_{1} \ldots x_{m}$, there exists a continuation $x$ which does not belong to the set $T$.

Indeed, as such a continuation, we can simply take a sequence

$$
x=x_{1} \ldots x_{m} x_{m+1} x_{m+2} \ldots
$$


where for $i>m$, we take $x_{i}=s_{\mathcal{P}, i}$ if $i \in S_{\mathcal{P}}$ and $x_{i}=0$ otherwise. For this new sequence, for every $N$, at most $m$ first instances may lead to results different from $s_{\mathcal{P}, i}$, so we have

$$
\#\left\{i \leq N: i \in S_{\mathcal{P}} \& x_{i}=s_{\mathcal{P}, i}\right\} \geq \#\left\{i \leq N: i \in S_{\mathcal{P}}\right\}-m .
$$

When $N \rightarrow \infty$, then $\#\left\{i \leq N: i \in S_{\mathcal{P}}\right\} \rightarrow \infty$, so for sufficiently large $N$, we have

$$
\#\left\{i \leq N: i \in S_{\mathcal{P}}\right\}-m>(1-\varepsilon) \cdot \#\left\{i \leq N: i \in S_{\mathcal{P}}\right\},
$$

thus,

$$
\#\left\{i \leq N: i \in S_{\mathcal{P}} \& x_{i}=s_{\mathcal{P}, i}\right\}>(1-\varepsilon) \cdot \#\left\{i \leq N: i \in S_{\mathcal{P}}\right\},
$$

and we cannot have

$$
\#\left\{i \leq N: i \in S_{\mathcal{P}} \& x_{i}=s_{\mathcal{P}, i}\right\} \leq(1-\varepsilon) \cdot \#\left\{i \leq N: i \in S_{\mathcal{P}}\right\} .
$$

Therefore, this continuation does not belong to the set $T$.

$3.4^{\circ}$. Finally, since the formula (1) explicitly defines the set $T$, this set $T$ is clearly definable.

So, $T$ is a physical theory, hence $\omega \notin T$, and the proposition is proven. 